The Israeli Journal of Aquaculture - Bamidgeh, IJA_69.2017.1417, 10 pages

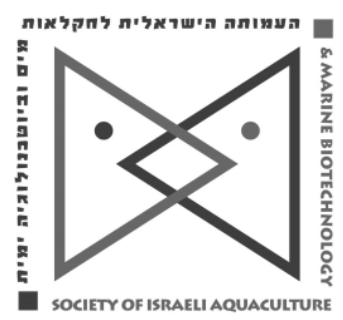

The IJA appears exclusively as a peer-reviewed on-line open-access journal at http://www.siamb.org.il. To read papers free of charge, please register online at registration form.

Sale of IJA papers is strictly forbidden.

\title{
Molecular Characterization and Possible Immune Function of Two Members of Interleukin Family from Trachinotus ovatus
}

\section{Kecheng Zhu ${ }^{1,2,3}$, Huayang Guo ${ }^{1,3}$, Nan Zhang ${ }^{1,3}$, Shigui Jiang ${ }^{1,2,3}$, Dianchang Zhang ${ }^{1,2,3, *}$}

${ }^{1}$ Key Laboratory of South China Sea Fishery Resources Exploitation and Utilization, Ministry of Agriculture, South China Sea Fisheries Research Institute, Chinese Academy of Fishery Sciences, 231 Xingang Road West, Haizhu District, Guangzhou 510300,China

${ }^{2}$ Engineering Technology Research Center, Guangdong Province, China

${ }^{3}$ Key Labaoratory of Fishery Ecology \& Environment, Guangdong Province, China

Keywords: Trachinotus ovatus; interleukin-7 (IL7) and interleukin-8 (IL8); Photobacterium damselae; gene expression.

\begin{abstract}
Interleukins (ILs) are a group of cytokines which play a core regulatory role in the immune system. In the present study, two members of the IL family, IL7 and IL8, were detected in Trachinotus ovatus. IL7 and IL8 cDNAs of T. ovatus consist of a 492 bp and 300 bp ORF (open reading frame) encoding a polypeptide of 163 and 99 amino acids, respectively. Multiple sequence alignment revealed that IL7 and IL8 contain characteristic arrangements of several conserved cysteine residues, which in $T$. ovatus are in positions $20,57,67,105,140,152$ and 35, 37, 61, 78, respectively. The phylogenetic tree showed that all ILs fell into four categories. Moreover, IL7 and IL8 mRNA of T. ovatus were constitutively expressed at different levels in all examined tissues, except muscle. Transcripts of IL7 were mainly expressed in liver, intestine, kidney, stomach, and fin, while transcripts of IL8 were highly detected in the eye, liver, kidney, and intestine of healthy fish. After Photobacterium damselae innoculation, MRNA levels of IL7 were higher than IL8 in the spleen and intestine, however, mRNA expression levels of IL7 were lower than IL8 in kidney $3 \mathrm{~h}$ post-injection. These results suggest that the two IL molecules play an important role in the inflammatory response of $T$. ovatus.
\end{abstract}

\footnotetext{
* Corresponding author. Dr. Dian C. Zhang; Email address: zhangdch@scsfri.ac.cn
} Tel.: +86 02089108316; fax: +8602089022702 


\section{Introduction}

Interleukins (ILs) belong to a sub-cluster of cytokines, which are involved in the intercellular regulation of the immune system. It was initially thought that ILs transmitted signals only between leucocytes, but it is now known that they are produced by, and target, various cells as well, and are composed of a complex system of cellsignalling within the immune system. Since the first description of ILs more than 30 years ago (Gery et al., 1972), 37 ILs were discovered. Most researchers have been concerned with these in mammals, and only recently has interest been extended to lower vertebrates, including fish (Pérez-Cordón et al., 2014).

ILs are divided into six families. Eleven members of the mammalian IL-1 family include: IL-1a(IL-1F1), IL-1 $(\mathrm{IL}-1 \mathrm{~F} 2), \mathrm{IL}-1$ receptor antagonist (IL-1ra/IL-1F3), IL-18 (IL-1F4), IL-1F5-10 and IL-33 (IL-1F11), only IL-1ß. IL-18 has now been found in fish (Secombes et al., 2011). The IL-2 subfamily of cytokines includes IL-2/4/7/9/15/21. All these molecules exist in fish except for IL-9. IL-10 is an anti-inflammatory cytokine, and a member of the class II cytokine family that includes IL-10/19/20/22/24/26 and the interferons (Lutfalla et al., 2003), IL-10/20/22/26 that have also been studied in fish (Secombes et al., 2011). Interleukin-7 is essential for lymphocyte development and plays a central role in the survival, proliferatio, and maturation of T and B cells (Namen et al., 1988). Moreover, interleukin-8 production has been observed in a wide variety of cells, including monocytes, $\mathrm{T}$ lymphocytes, neutrophils, vascular endothelial cells, and hepatocytes (Wang et al., 2013).

There is limited information about IL7 in fish. Takifugu rubripes IL7 was cloned and showed constitutive expression in head kidney, spleen, liver, intestine, gill, and muscle, with increased expression in head kidney cultures stimulated with Lipopolysaccharide (LPS), Poly I:C, or Phytohemagglutinin (PHA) (Kono et al., 2008). The anti-inflammatory profile at 64 days of incubation with Enteromyxum leei was represented mainly by the significant up-regulation of IL7 in the posterior intestine of Sparus aurata (PérezCordónet al., 2014). Furthermore, IL8 was a prototypical CXC chemokine (Jimenez et al., 2006; Li and Yao, 2013). By recruiting immune cells to injury sites, chemokines play a vital role in both innate and adaptive immune responses (Esche et al., 2005). Most chemokines have four conserved cysteine residues which are important for their tertiary structure (Fernandez and Lolis, 2002; Joseph et al., 2010). IL8 signalling is induced by inflammatory signals (TNF-a and IL-1), reactive oxygen species, death receptors, and steroid hormones, accordingly leading to the activation of transcription factors such as NFkB, AP-1, HIF-1, and STAT3 (Campbell et al., 2013).

The silverfish Trachinotus ovatus (Linnaeus 1758), is widely cultured in the AsiaPacific region. The fish is a delicacy that has been favoured for its fast growth and high quality flesh, and is recognized as an important aquaculture species in China (Sun et al., 2014; Zhen et al., 2014). Nevertheless, death rate is high due to the pathogen Photobacterium damselae in T. ovatus (Su et al., 2012). To investigate the function of IL7 and IL8 in response to $P$. damselae, we analysed the sequence and expression characterization of IL7 and IL8 in liver, spleen, kidney, and intestine from $T$. ovatus. Additionally, sequence alignments of the IL family were also analysed. This research aims to improve understanding of the innate immune system after inoculating the fish with specific pathogens. And thereby reduce losses to farmers by finding a way to prevent damage caused by $P$. damselae.

\section{Materials and methods}

Fish rearing conditions and experimental design. Juvenile $T$. ovatus were obtained from Linshui Marine Fish Farm in Hainan Province, China. Before the initiation of the feeding trial the fish $(250 \pm 12.6 \mathrm{~g})$ were stored in a cement tank with re-circulating water. Fish were acclimated and fed the experimental diet for 7 days under a controlled photoperiod (14 h/day:10 h/night). The fish were randomly distributed into two groups (a) Control group and (b) Bacteria inoculated group with five replicates each. The experimental group was inoculated with $100 \mu \mathrm{L} P$. damselae resuspended in phosphate-buffered saline (PBS) to $10^{9} \mathrm{CFU} / \mathrm{mL}$ (Su et al., 2012) which was administered by intraperitoneal injection, while the control group was injected with PBS $(100 \mu \mathrm{L})$. During the experimental period, temperature of the pool water was $22 \pm 1^{\circ} \mathrm{C}$ and dissolved oxygen in the water was $>6 \mathrm{mg} / \mathrm{L}$. 
Sample collection. In order to study the effect of the bacteria in $T$. ovatus, fish from both control and treatment groups at $0,1,3,6,12,24,48$, and $72 \mathrm{~h}$ after inoculation, were anesthetized using MS222 (0.1 g/L; Sigma, Alcobendas, Spain). Liver, spleen, kidney and intestine were taken from all groups and immediately stored in RNA (Life Technologies, Waltham, MA, USA) reagent until used.

To detect the spatial expression of IL7 and IL8, tissue samples of adult T. ovatus ( $\mathrm{n}$ $=5$ ) including heart, stomach, brain, eye, gill, fin, skin, muscle, liver, spleen, kidney, intestine, and blood tissues were collected.

Total RNA isolation and formation of CDNA. Total RNA was isolated from different tissues using Trizol kit (Invitrogen, USA). The quality and quantity (concentration) of isolated RNA were determined by NANODROP 2000 spectrophotometer (Thermo, Scientific). Two micrograms of RNA and $0.5 \mu \mathrm{g}$ of Oligo $\mathrm{d}(\mathrm{T})_{16}$ were reacted for $5 \mathrm{~min}$ at $70^{\circ} \mathrm{C}$. After incubation for $2 \mathrm{~min}$ on ice, the mixture was reverse transcribed with 200 units of M-MLV reverse transcriptase (Promega), $5 \times$ buffer, 25 units RNasin and $0.8 \mathrm{mM}$ dNTPs in a total volume of $25 \mu \mathrm{L}$ and extended for $1 \mathrm{~h}$ at $42^{\circ} \mathrm{C}$. cDNA was then stored at $-20^{\circ} \mathrm{C}$ until used.

Gene cloning of IL7 and IL8. Full-length CDNA sequences of two ILs were obtained by transcriptome sequencing (unpublished), then sequences of intron and exon were verified using IL7 and IL8 primers (Table 1), respectively. The PCR products were ligated into a pGEM $\AA-T$ easy vector (Promega), respectively, and then sequenced on an ABI 3730XL Automated Sequencer using Sequencing Analysis 5.2.

Table 1. Primers used for cloning and expression.

\begin{tabular}{llll}
\hline Gene & Primer sequences $\left(5^{\prime}-3^{\prime}\right)$ & Product length(bp) & Application \\
\hline$I L-7-F 1$ & CAAAAGCCACAAGAACCG & \multirow{2}{*}{810} & ORF \\
$I L-7-R 1$ & CAAAACAGCCGCAAGAGA & & \\
$I L-7-F 2$ & AAGAATGACCAATGCCTC & \multirow{2}{*}{1263} & ORF \\
$I L-7-R 2$ & ACGCCTGTGAGACAAACT & & \\
$I L-8-F$ & GCAGTTAGTTTTACCG & \multirow{2}{*}{1087} & ORF \\
$I L-8-R$ & CTAAGGGAACACATTTGA & & Real-time \\
$I L-7-q R T-F$ & CACAACTCATCCTGCCCA & \multirow{2}{*}{184} & PCR \\
$I L-7-q R T-R$ & CGTTAGTCGGTTCCCAA & & Real-time \\
$I L-8-q R T-F$ & TCATTTCATTGCTGTGG & \multirow{2}{*}{118} & PCR \\
$I L-8-q R T-R$ & ATGGGTTGCTCTCTGTC & & Real-time \\
$E F-1 a-F$ & CCCCTTGGTCGTTTGCC & \multirow{2}{*}{100} & PCR \\
$E F-1 a-R$ & GCCTTGGTTGTCTTCCGCTA & & \\
\hline
\end{tabular}

Bioinformatics analysis. IL7 and IL8 of nucleotide and amino acid sequence similarity searches were performed using the BLAST program (http://blast.ncbi.nlm.nih.gov/Blast.cgi). The Open Reading Frame (ORF) Finder (http://www.ncbi.nlm.nih.gov/projects/gorf/) was used to predict the coding sequence of IL7 and IL8. Molecular weight (Mw), theoretical isoelectric point (pI) and features of the protein were obtained referring to the ExPASy analysis system (http://us.expasy.org/tools), and the SignaIP 4.1 Server was used for signal peptide prediction (http://www.cbs.dtu.dk/services/). Multiple sequences were aligned using the Clustal X. program (Julie et al., 2006), and phylogenetic trees were constructed by MEGA 5.1 software using the unweighted pair-group method with arithmetic means (UPGMA) method (Kumar et al., 2006).

Spatial and temporal expression analysis of IL7 and IL8. IL7 and IL8 mRNA expression was analysed by quantitative real-time PCR (qRT-PCR) (Bustin et al., 2009). Specific primer pairs for IL7 and IL8 and the reference gene EF-1a (elongation factor 1, alpha) were designed (Table 1). qRT-PCR was performed in $20 \mu \mathrm{L}$ total volume containing $10 \mu \mathrm{L}$ SYBR Green qPCR Master Mix (Toyobo, Osaka, Japan), $0.3 \mu \mathrm{M}$ of each primer, $5 \mu \mathrm{L}$ RNase-free $\mathrm{H}_{2} \mathrm{O}$, and $2 \mu \mathrm{L}$ cDNA. The qRT-PCR program consisted of an initial denaturation at $95^{\circ} \mathrm{C}$ for $3 \mathrm{~min}$, followed by 40 cycles of amplification for $7 \mathrm{~s}$ at $95^{\circ} \mathrm{C}, 10$ $\mathrm{S}$ at specific annealing temperatures (Table 1 ), $15 \mathrm{~s}$ at $72^{\circ} \mathrm{C}$, and final extension for 10 min at $72^{\circ} \mathrm{C}$ in a Light Cycler $\AA 480$ II (Roche, Basel, Switzerland). Relative expression was determined using the $2^{-\triangle \Delta C T}$ method (Livak and Schmittgen 2001). 
Statistical analysis. All data from the relative mRNA expression represented at least three replications along with means \pm standard error of the mean (SE). Statistical analysis of difference was performed by using SPSS 19.0 (SPSS Inc., Chicago, IL, USA). Differences were considered significant at the $p<0.05$ level.

\section{Results}

Cloning and sequence analysis of T. ovatus IL7 and IL8. Two fragments of 3072 and 822 bp were amplified by $I L 7-F / R$ and IL8-F/R, respectively (Table1). IL7 cotained 4 exon and 3 intron, while IL8 cotained 3 exon and 3 intron. After alignment of the two fragments, we found that the CDNA of T. ovatus IL7 [GenBank accession no. KT922005] and IL8 [GenBank accession no. KT922006] consisted of a 492 bp and 300 bp open reading frame encoding a polypeptide of 163 and 99 amino acids, respectively. The predicted IL7 and IL8 proteins have a molecular mass of $18 \mathrm{kDa}$ and $11 \mathrm{kDa}$, and an isoelectric point of 9.35 and 8.82 , respectively. SignaIP 4.1 analysis showed that the signal peptide existed in both of the two IL amino acid sequences.

Analysis of the deduced amino acid sequence. Multiple sequence alignment revealed that the two ILs contained a typical arrangement of several conserved cysteine residues as found in other species (Fig.1). Moreover, IL8 had a highly conserved ELR motif which is replaced by tripeptide substitutions in most teleost fish studied, such as EQH, ELH, EMH or ELR in mammals. T. ovatus IL8 has an EQH motif like T. rubripes IL8 homologue (Fig. 1B).

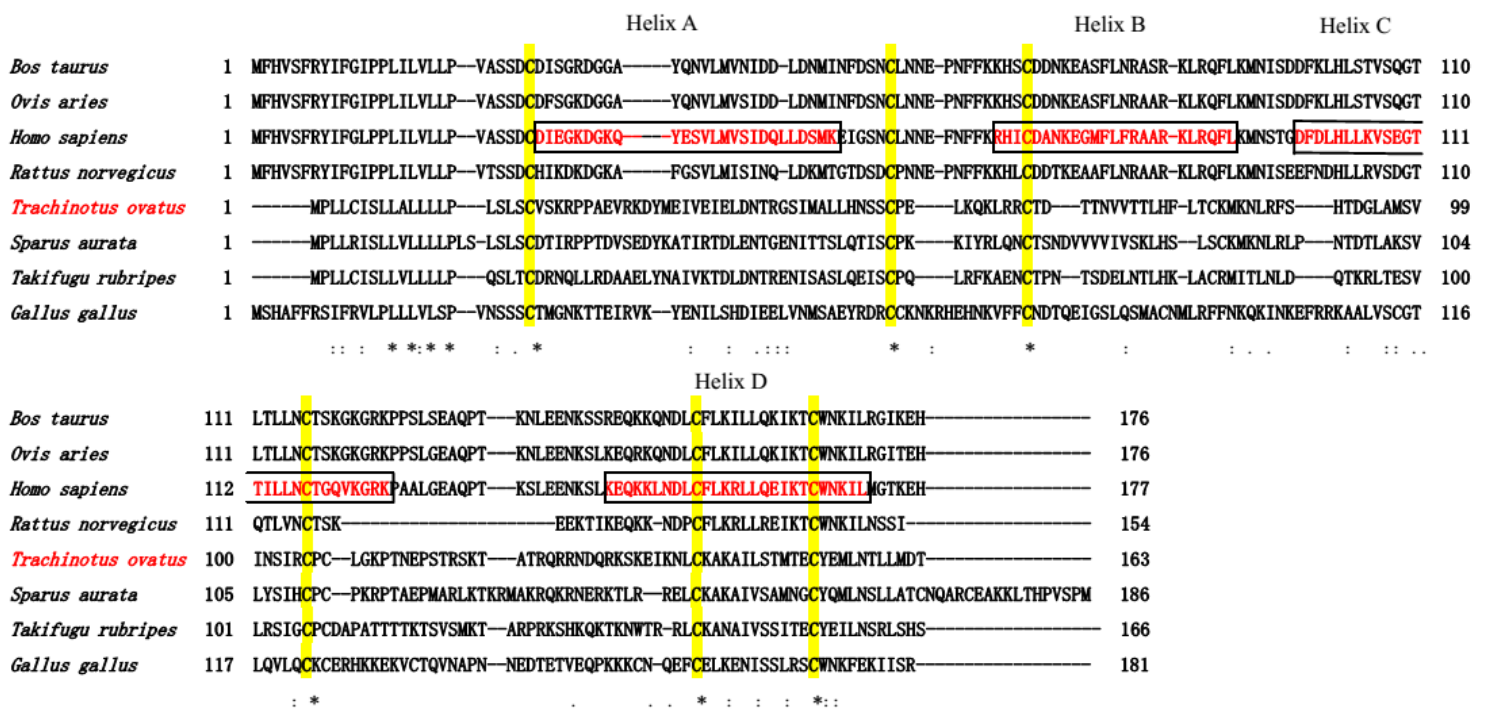

Fig. 1A.

\begin{tabular}{|c|c|c|c|}
\hline Trachinotus ovatus & 1 & KISRVIVIAVVVLLASLTVSEGMSLRSLGM & QRCIQTESKPIG-RHIEKVELIPANSHCEETEIIATLKKTGQEVCLDPEAPWVKRVIEKILSNRRR- \\
\hline Oplegnathus fasciatus & 1 & D. & QTESKPIG-RHIGKVELIPANSHCEETEIIATLKKTGQEVCLDPEAPWVKKVIKAIMNKSR- \\
\hline Thunnus maccoyii & 1 & MMSSKVTVTAVVVLLCLLATSEVMSLTSLGV & ITESKPIG-RHIQKVELIPANSHCEETEIIATLKKTGQEVCLDPEAPWVKKVIKKILSNRS- \\
\hline Latris lineata & 1 & MKSSRVIVTSIVVLLAFLAISEGMSLRSLGV & QTESKPIG-RHIGKVELIPANSHCEETEIIATLKKTGQEVCLDPEAPWVKRVIQKILSNRRR- \\
\hline Siniperca chuatsi & 1 & MKSSRVIITSIVVLLAFLAISEGMSLRSLGV & TESKPIG-RHIGKVELIPANSHCEETEIIATLKKTGQEVCLDPEAPWVKRVIQKIMSNKRR—- \\
\hline Dicentrarchus labrax & 1 & MMSSKVFATSIVVLLAFLAISEGMSLRS & TESKPIG-RHIGKVELIPANSHCEETEIIATLKKTGQEVCLDPEAPWVKKVIQKILSNRRR- \\
\hline Sparus aurata & 1 & MMSSRVFIVTI & QTERKPIG-RHIEKVELIPASSHCEETEIIATLKRTGQEVCLDPEAPWVKKVIQRILSNARR— \\
\hline Lateolabrax japonicus & 1 & MKSSRVIVTSIVVLLAFLAITEGMSLRSLGV & TESKPIG-RHIEKVELIPANSHCEETEIIATLKKTGQEVCLDPEAPWVKKVINKILSNRTP- \\
\hline Lutjanus sanguineus & 1 & MMSSRVFIASIVVLLAFLATSEGMSLGSPGV & QTESRPIG-RHIGKVELILPNSHCEDTEIIATLKKTGQEVCLDPEAPWVKKVIERIMTN--- \\
\hline Takifugu rubripes & 1 & SNGMSLRSLGV & QTESRPIG-RHIGKVELIPPNSHCEETEIIATLKMSGQEVCLDPKAPWVKKVINKIMSSRQR一- \\
\hline omis niloticus & 1 & MISNRIIVSSIVVLLALFATCEG----MGV & IKTESKPIG-RHIEKVELILPNSHCEETEIIATLKRTGEEVXLNPEAPWVKKVINKIMSSRS- \\
\hline Maca & 1 & -MTSKLAVALLAAFLLSAALCEGAVLPRS & KTYSKPFHPKFIKELRVIESGPHCANTEIIVKLSD-GRELCLDPKEPWVQRVVEKFVKRAENQNP \\
\hline Homo $s$ & 1 & -MTSKLAVALLAAFLISAALCEGAVLP & VQRVVEKFLKRAENS-- \\
\hline
\end{tabular}

Fig. 1B.

Figs. 1A \& B. Comparison of deduced amino acid sequences of Trachinotus ovatus IL7 (A) and IL8 (B) with published IL7 and IL8 in other species, respectively. The predicted signal peptide is underlined and the a-helix regions in the human molecule are indicated by box areas (A) (Kono et al., 2008). The ELR motif is boxed (B). Cysteine residues, important in forming disulfide bonds, are yellow. Dashes represent gaps created to maximize the degree of identity among all compared sequences. The accession numbers of the IL7 and IL8 sequences used are listed in table 2 and 3, respectively. 
These results suggested that the $T$. ovatus IL7 and IL8 proteins probably had similar functions as those observed in mammalian counterparts. Furthermore, T. ovatus IL7 and IL8 had the highest homology with S. aurata IL7 (Table 2) and Latris lineate IL8 (Table $3)$, respectively.

Table 2. Trachinotus ovatus IL7 used in multiple alignment.

\begin{tabular}{lllll}
\hline Species & Protein name & GenBank No. & Amino acids & Identities (\%) \\
\hline Trachinotus ovatus & IL7 & KT922005 & 163 & 100 \\
Sparus aurata & IL7 & AGS55342.1 & 186 & 47 \\
Takifugu rubripes & IL7 & CAP05285.1 & 166 & 45 \\
Gallus gallus & IL7 & CAP62363.1 & 181 & 26 \\
Bos taurus & IL7 & NP_776349.1 & 176 & 22 \\
Ovis aries & IL7 & NP_001009777.1 & 176 & 18 \\
Homo sapiens & IL7 & NP_000871.1 & 177 & 16 \\
Rattus norvegicus & IL7 & NP_037242.2 & 154 & 12 \\
\hline
\end{tabular}

Table 3. Trachinotus ovatus IL8 used in multiple alignment.

\begin{tabular}{lllll}
\hline Species & Protein name & GenBank No. & Amino acids & Identities (\%) \\
\hline Trachinotus ovatus & IL8 & KT922006 & 99 & 100 \\
Latris lineata & IL8 & ACQ99511.1 & 99 & 89 \\
Thunnus maccoyii & IL8 & AGH24760.1 & 99 & 88 \\
Lateolabrax japonicus & IL8 & ACK57558.1 & 99 & 88 \\
Oplegnathus fasciatus & IL8 & ADK35757.1 & 99 & 87 \\
Siniperca chuatsi & IL8 & AKA66316.1 & 99 & 86 \\
Dicentrarchus labrax & IL8 & CAM32186.1 & 99 & 86 \\
Takifugu rubripes & IL8 & NP_001027759.1 & 98 & 78 \\
Sparus aurata & IL8 & AGS55343.1 & 95 & 75 \\
Lutjanus sanguineus & IL8 & AGV99968.1 & 96 & 76 \\
Oreochromis niloticus & IL8 & NP_001266633.1 & 93 & 70 \\
Macaca mulatta & IL8 & NP_001028137.1 & 101 & 45 \\
Homo sapiens & IL8 & NP_001028137.1 & 99 & 43 \\
\hline
\end{tabular}

The amino acid sequences of 181 bilateria ILs were used to construct a multiple sequence alignment. In order to evaluate the molecular evolutionary relationship of ILs, a phylogenetic tree was generated by the WAG model with UPGMA method within the MEGA 5.1 software package is displayed in Fig. 2.

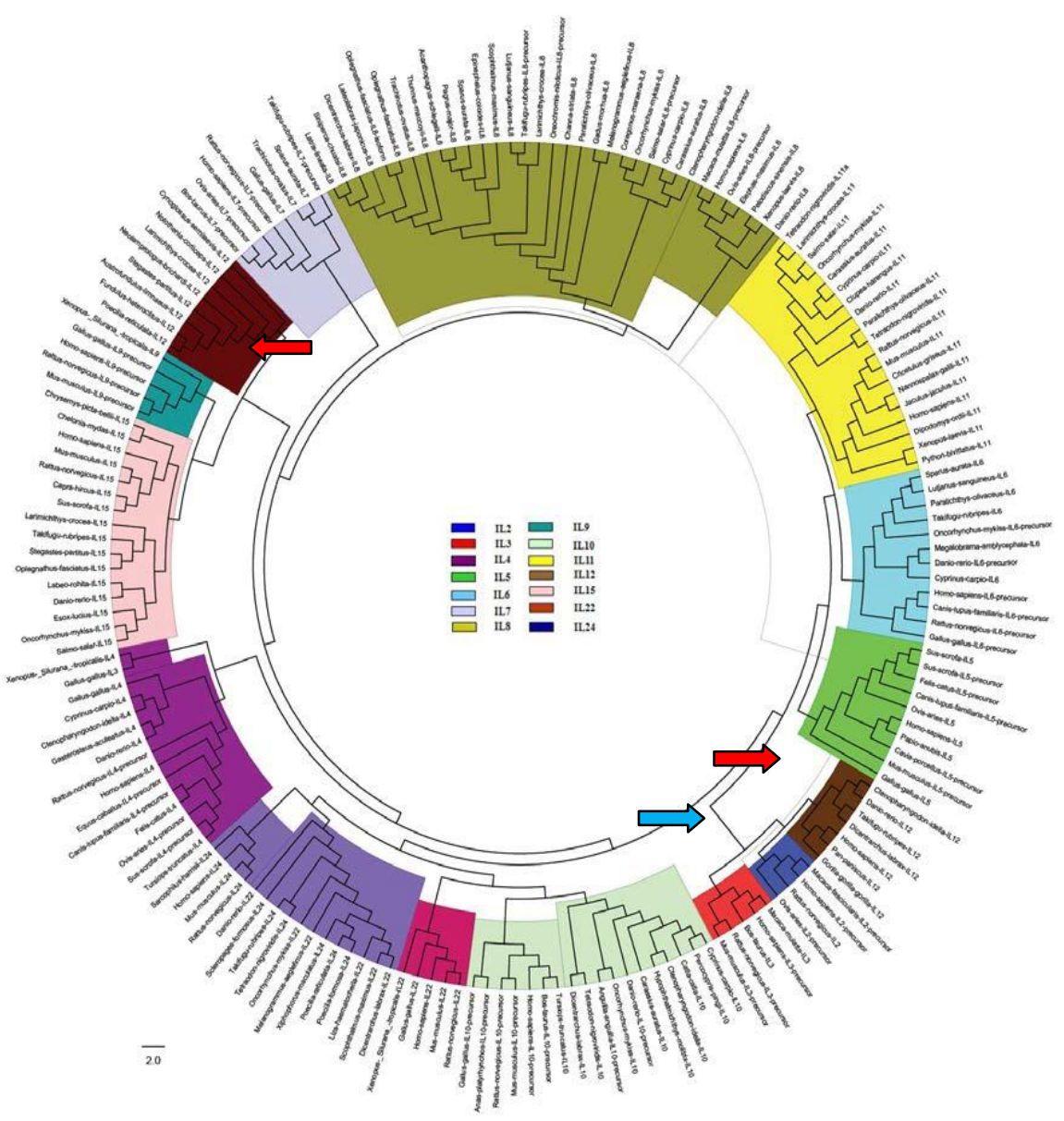

Fig. 2. Phylogenetic tree analysis of ILs family in vertebrates. Sequence alignment of ILs was analyzed using the MEGA 5.1 software with unweighted pairgroup method with arithmetic means (UPGMA) method. The IL7 and IL8 are indicated by the white and army green font, respectively. IL2 and IL3 was show by blue arrows, while IL12A and IL12B was show by red arrows. The accession numbers of the sequences used in the phylogenetic analysis are listed in supplement table. 
All ILs fell into four categories which were interleukin-2 subfamily (IL4/7/8/9/12B/15), interleukin-10 subfamily (IL10/22/24), heterodimeric interleukins (IL12A) and others (IL2/3/5/6/11). Fig.2 shows that IL8 clustered into the interleukin-2 subfamily subgroup. Interesting, IL2, IL3 and IL12A formed a cluster, while IL12B fell into interleukin-2 subfamily.

Tissue expression analysis of IL7 and IL8 mRNA. Spatial expression patterns revealed that both IL7 and IL8 were constitutively expressed in a wide range of tissues except muscle in healthy fish. IL7 transcripts were predominantly detected in liver, intestine, kidney, stomach, and fin tissue, but marginally detected in brain, eye, gill, and spleen tissue (Fig. 3A). Meanwhile, for IL8, the highest transcript expression was observed in the eyes followed by liver, kidney, and intestine tissue, while low transcript expression was observed in gill and spleen tissue (Fig. 3B).

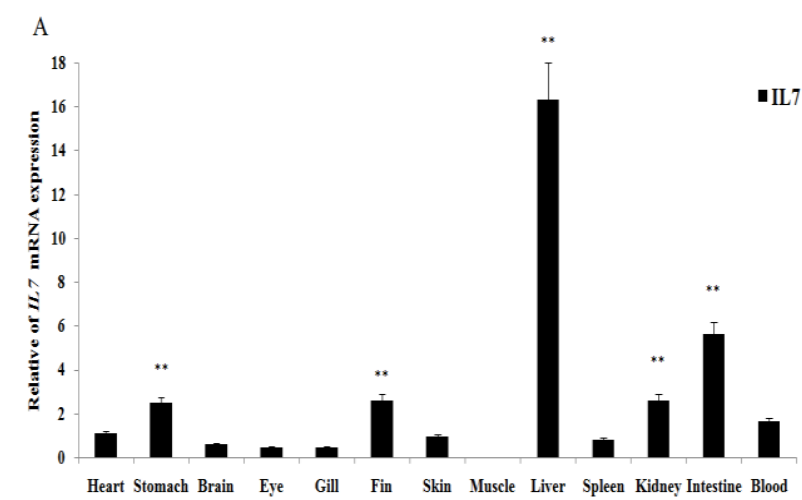

Fig. 3A.

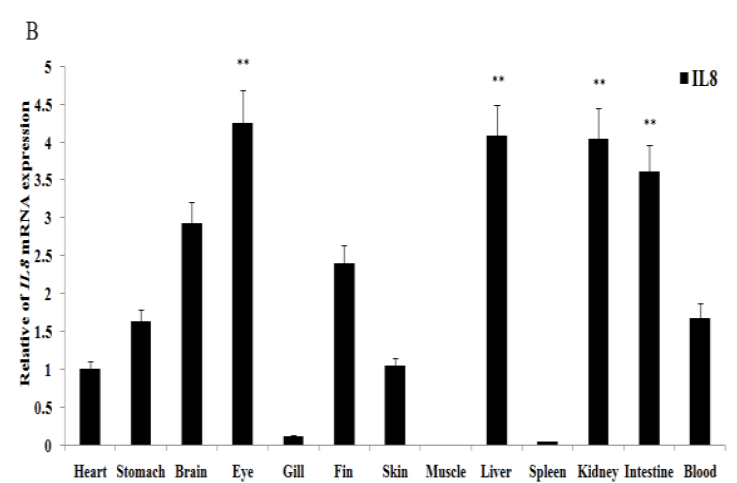

Fig. 3.B

Fig. 3. Gene transcriptions of $I L 7(A)$ and $I L 8$ (B) in various tissues of $T$. ovatus. Significant differences at $P<0.01$ are labeled with $* *$, mean \pm SEM of each mRNA quantity is shown for each of the tissues tested.

IL7 and IL8 expression after bacterial inoculation. To understand the modulation of $T$. ovatus IL7 and IL8 expression upon P. damselae induction, we conducted qRT-PCR on liver, spleen, kidney, and intestine tissues to assess T. ovatus IL7 and IL8 mRNA levels during $72 \mathrm{~h}$ stimulation. Time course analysis of gene transcription showed that at $3 \mathrm{~h}$ post- $P$. damselae inoculation, both $T$. ovatus IL7 and IL8 transcription was rapidly down-regulated to the lowest level in liver and spleen tissue, then quickly increased and reached peak levels at $24 \mathrm{~h}$ (3.23-fold higher) and $6 \mathrm{~h}$ (2.57-fold higher), respectively, subsequently they became down-regulated (Fig.4A and B). However, IL8 mRNA quickly decreased from $3 \mathrm{~h}$ to $72 \mathrm{~h}$ in liver tissue (Fig.4A). Moreover, P. damselae challenge upregulated IL7 expression at $1 \mathrm{~h}$ until peak levels were achieved at $72 \mathrm{~h}, 72 \mathrm{~h}$, and $48 \mathrm{~h}$ in spleen, kidney, and intestine tissue, respectively (Fig.4B, C and D). A similar transcription pattern of IL8 was observed in kidney and intestinal tissue (Fig.4C and D).

After $3 \mathrm{~h}$ bacterial challenge, the mRNA levels of IL7 were dramatically higher than IL8 in spleen and intestine (Fig.4B and D), nevertheless, expression was lower in IL7 than IL8 in kidneys (Fig.4C).
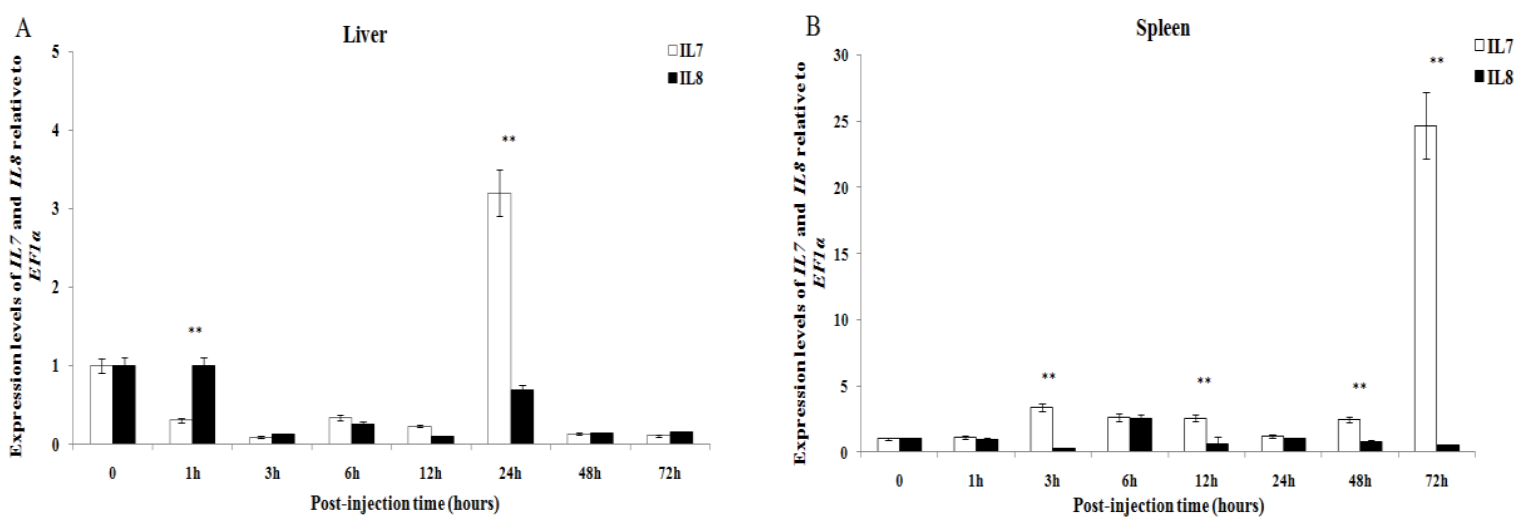

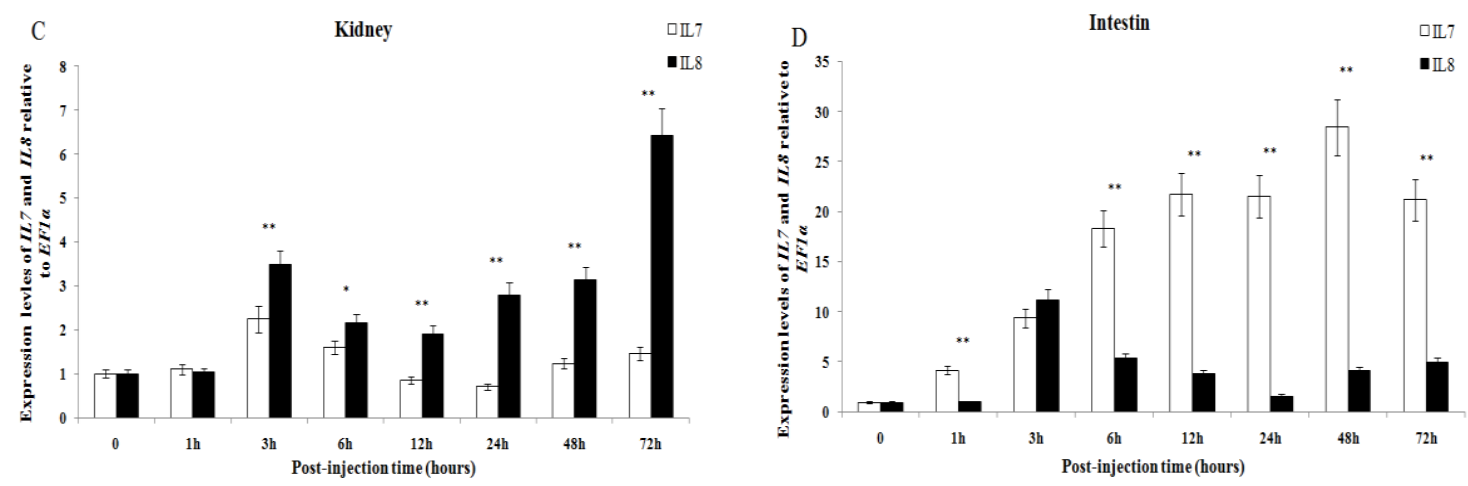

Fig. 4. Temporal expression of IL7 and IL8 in liver (A), spleen (B), kidney (C), and intestine (D) after bacterial inouclation with Photobacterium damselae for the indicated times. EF-1a expression was used as an internal control for real-time PCR. Significant differences at $P<0.01$ are labeled with $* *$, mean \pm SEM of each mRNA quantity is shown for each tissues tested.

\section{Discussion}

The term IL was first introduced in 1972 referring to T-lymphocytes. It is now known that 37 ILs are produced by a wide variety of cell types (Gery et al., 1972; Pérez-Cordón et al., 2014), and many are synthesized by macrophages/monocytes, CD4 ${ }^{+} \mathrm{T}$ helper cells, and endothelial cells (Secombes et al., 2011). Recently, several cytokine genes have been researched in teleosts, such as T. rubripes IL7 (Kono et al., 2008), S. aurata IL7 (Perez-Cordon et al., 2014), Paralichthys olivaceus IL8 (Zhao et al., 2015), and Larimichthys crocea IL8 (Mu et al., 2015). Here we identified a T. ovatus IL7 that shared low identity to previously reported species (S. aurata $47 \%$, T. rubripes $45 \%$ ) (Table 2). Two cysteines ( $\mathrm{Cys}^{59}$ and $\mathrm{Cys}^{154}$ ) are involved in the formation of a disulphide bond in humans (Kono et al., 2008). Moreover, there are six cysteines which are strictly conserved and essential for the biological activity of mammalian IL7 (Cosenza et al., 1997). They are also conserved in T. rubripes $\left(\mathrm{Cys}^{20}, \mathrm{Cys}^{57}, \mathrm{Cys}^{67}, \mathrm{Cys}^{106}, \mathrm{Cys}^{143}, \mathrm{Cys}^{155}\right.$ ) and T. ovatus $\left(\mathrm{Cys}^{20}, \mathrm{Cys}^{57}, \mathrm{Cys}^{67}, \mathrm{Cys}^{105}, \mathrm{Cys}^{140}, \mathrm{Cys}^{152}\right.$ ) IL7 molecules. The T. ovatus IL7 is predicted to form these disulphide bonds which may indicate a similar receptor binding, activity and secondary structure to other vertebrates. Type I cytokines form four a-helical bundle structures (Bazan, 1990). Mutational analysis showed that Lys ${ }^{145}$, Leu ${ }^{160}$, Lys $^{164}$, and $\operatorname{Trp}^{167}$ in helix $D$ are required to stimulate IL7-dependent pre-B-cell proliferation. It was suggested that helix $\mathrm{D}$ is important for receptor activation and biological activity (Kono et al., 2008). Nevertheless, the only residue that is absolutely conserved in this domain and in all the mammalian IL7, T. rubripes (Lys ${ }^{134}$ ) and T. ovatus $\left(\right.$ Lys $^{133}$ ) IL7 are the same as the human Lys ${ }^{145}$. This may indicate that this residue plays an important role in the biological activity of IL7 (Kono et al., 2008).

We also observed that $T$. ovatus IL8 shared a high homology to the fish IL8 $(70 \%-89 \%)$. The ELR motif is a conserved feature of the mammalian and avian IL8 sequence (Wang et al., 2013). Moreover, human ELR-positive CXC chemokines usually recruit neutrophils and other polymorphonuclear leukocytes (Strieter et al., 1995; Wang et al., 2013). In fish, the ELR motif was only found in M. aeglefinus and G. morhua IL8, T. ovatus IL8 has an EQH motif (Fig. 1B). However, without the ELR motif in CXC chemokines, some fish recombinant proteins still attracted neutrophils (Huising et al., 2003; Hu et al., 2011; Sun et al., 2011; Wang et al., 2013).

T. ovatus IL7 mRNA was constitutively expressed in all tissues examined except in the muscle, with the highest levels in the liver and intestine (Fig.3). These results were consistent with those reported in T. rubripes IL7, where the IL7 gene was expressed in several tissues, exhibiting the strongest expression in the intestine and head kidney (Kono et al., 2008). IL8 mRNA was detected at high levels in kidney, liver, and intestine (gut) tissue (Laing et al., 2002; Huising et al., 2003; Corripio-Miyar et al., 2007; van der Aa et al., 2010; Hu et al., 2011; Sun et al., 2011; Tamura et al., 2013), in agreement with the findings of high to moderate expressions of IL8 in various tissues of $T$. ovatus. 
Ictalurus punctatus IL8 mRNA was absent in liver, muscle, skin, and heart tissue which indicated distinct expression patterns for IL8 in different fish species (Chen et al., 2005). The varying tissue expression patterns of IL8 might be due to differences in the fish species or physiological status (Mu et al., 2015).

To further study the expression of IL7 within the immune system of fish, the expression pattern of the T. ovatus IL7 and IL8 genes were analysed in liver, spleen, kidney, and intestine stimulated with $P$. damselae. The results indicated that both IL7 and IL8 mRNA levels were significantly up-regulated in spleen, kidney, and intestine of $T$. ovatus injected with $P$. damselae. This indicates that IL7 and IL8 are involved in the immune response against $P$. damselae infection. This was similar to the results obtained in $T$. rubripes, where IL7 mRNA levels were increased in head kidney cells after LPS, poly I:C and PHA stimulation (Kono et al., 2008). IL8-L1 mRNA was up-regulated in spleen and head kidney tissues of $L$. crocea after stimulation with bacterial vaccine (Mu et al., 2015) and also with Vibrio parahaemolyticus and LPS (Li \& Yao, 2013). The common carp IL8-2 mRNA increased after stimulation of PBLs with LPS (Van der Aa et al., 2010). P. olivaceus IL8 expression levels were up-regulated in the kidneys after $S$. iniae infection (Zhao et al., 2015). Therefore, the remarkable increases of IL7 and IL8 expression by pathogenic bacteria, bacterial product LPS, and double-stranded viral RNA (Poly I:C) stimulation, strongly support its potential in pro-inflammatory function (Mu et al., 2015).

IL7 expression was significantly higher in spleen and intestine than IL8 expression after a $3 \mathrm{~h}$ bacterial challenge, which indicates that IL7 showed a more sensitive $P$. damselae induction in the spleen and intestine than IL8. In conclusion, the rapid increase in IL7 and IL8 expression by P. damselae stimulation suggests that they play roles in the inflammatory response. These results will provide a helpful foundation for the treatment of $P$. damselae infection.

\section{Conflict of interest}

The authors declare that they have no conflict of interest in this publication.

\section{Acknowledgements}

This research was funded by Central Public-interest Scientific Institution Basal Research Fund, CAFS (NO. 2016HY-JC0304), the Fishing Port Construction and Fishery Development Special Funds for Guangdong Province (Sci-tech Popularization, 2017A0008), the Special Scientific Research Funds for Central Non-profit Institute, Chinese Academy of Fishery Sciences (2015YD01, 2016A11JC03), and National Infrastructure of Fishery Germplasm Resources Project (2017DKA30407).

\section{References}

Bazan, J.F., 1990. Haemopoietic receptors and helical cytokines. Immunol Today 11, 350-354.

Bustin, S.A., Benes, V., Garson, J.A., Hellemans, J., Huggett, J., Kubista, M., et al., 2009. The MIQE guidelines: minimum information for publication of quantitative realtime PCR experiments. Clin. Chem. 55, 611-622.

Campbell, L.M., Maxwell, P.J., Waugh, D.J., 2013. Rationale and means to target proinflammatory interleukin-8 (CXCL8) signaling in cancer. Pharmaceuticals, 6, 929-959.

Chen, L., He, C., Baoprasertkul, P., Xu, P., Li, P., Serapion, J., et al., 2005. Analysis of a catfish gene resembling interleukin-8: cDNA cloning, gene structure, and expression after infection with Edwardsiella ictaluri. Dev. Comp. Immunol. 29, 135-142.

Corripio-Miyar, Y., Bird, S., Tsamopoulos, K., Secombes, C.J., 2007. Cloning and expression analysis of two pro-inflammatory cytokines, IL-1 beta and IL-8, in haddock (Melanogrammus aeglefinus). Mol. Immunol. 44, 1361-1373.

Cosenza, L., Sweeney, E., Murphy, J.R., 1997. Disulfide bond assignment in human interleukin-7 by matrix assisted laser desorption/ionization mass spectroscopy and sitedirected cysteine to serine mutational analysis. J Biol. Chem. 272, 32995-33000.

Esche, C., Stellato, C., Beck, L.A., 2005. Chemokines: key players in innate and adaptive immunity. J. Invest. Dermatol. 125, 615-628. 
Fernandez, E.J., Lolis, E., 2002. Structure, function, and inhibition of chemokines. Annu. Rev. Pharmacol. Toxicol. 42, 469-499.

Gery, I., Gershon, R.K., Waksman, B.H., 1972. Potentiation of T-lymphocyte response to mitogens. I. The responding cell. J Exp. Med. 136, 128-142.

Hu, Y.H., Chen, L., Sun, L., 2011. CXCL8 of Scophthalmus maximus: expression, biological activity and immunoregulatory effect. Dev. Comp. Immunol. 35, 1032-1039.

Huising, M.O., Stolte, E., Flik, G., Savelkoul, H.F., Verburg-van Kemenade, B.M., 2003. CXC chemokines and leukocyte chemotaxis in common carp (Cyprinus carpio L.). Dev. Comp. Immunol. 27, 875-888.

Jimenez, N., Coll, J., Salguero, F.J., Tafalla, C., 2006. Co-injection of interleukin 8 with the glycoprotein gene from viral haemorrhagic septicemia virus (VHSV) modulates the cytokine response in rainbow trout (Oncorhynchus mykiss). Vaccine 24, 5615-5626.

Joseph, P.R.B., Sarmiento, J.M., Mishra, A.K., Das, S.T., Garofalo, R.P., Navarro, J., et al., 2010. Probing the role of CXC motif in chemokine CXCL8 for high affinity binding and activation of CXCR1 and CXCR2 receptors. J. Biol. Chem. 285, 29262-29269.

Kono, T., Bird, S., Sonoda, K., Savan, R., Secombes, C.J., Sakai, M., 2008. Characterization and expression analysis of an interleukin-7 homologue in the Japanese pufferfish, Takifugu rubripes. FEBS J. 275, 1213-1226.

Kumar, S., Nei, M., Dudley, J., Tamura, K. 2006. MEGA: molecular evolutionary genetics analysis software for microcomputer. Bioinform. J. 10, 89-191.

Laing, K.J., Zou, J.J., Wang, T., Bols, N., Hirono, I., Aoki, T., et al., 2002. Identification and analysis of an interleukin 8 -like molecule in rainbow trout Oncorhynchus mykiss. Dev. Comp. Immunol. 26, 433-444.

Li, C., Yao, C.L., 2013. Molecular and expression characterizations of interleukin-8 gene in large yellow croaker (Larimichthys crocea). Fish Shellfish Immunol. 34, 799-809.

Livak, K.J., Schmittgen, T.D., 2001. Analysis of relative gene expression data using real-time quantitative PCR and the 2- $\Delta \Delta C T$ method. Methods 25, 402-408.

Lutfalla, G., Crollius, H.R., Stange-thomann, N., Jaillon, O., Mogensen, K., Monneron, D., 2003. Comparative genomic analysis reveals independent expansion of a lineage-specific gene family in vertebrates: the class II cytokine receptors and their ligands in mammals and fish. BMC Genomics 4, 29.

Mu, Y.N., Wang, K.R., Ao, J.Q., Chen, X.H., 2015. Molecular characterization and biological effects of a CXCL8 homologue in large yellow croaker (Larimichthys crocea). Fish Shellfish Immunol. 44, 462-470.

Namen, A.E., Lupton, S., Hjerrild, K., Wignall, J., Mochizuki, D.Y., Schmierer, A., Mosley, B., March, C.J., Urdal, D. \& Gillis, S., 1988. Stimulation of B-cell progenitors by cloned murine interleukin-7. Nature 333, 571-573.

Perez-Cordon, G., Estensoro, I., Benedito-Palos, L., Calduch-Giner, J.A., SitjaBobadilla, Perez-Sanchez, A.J., 2014. Interleukin gene expression is strongly modulated at the local level in a fish-parasite model. Fish Shellfish Immunol. 37, 201208.

Secombes, C.J., Wang, T., Bird, S., 2011. The interleukins of fish. Dev. Comp. Immunol. 35, 1336-1345.

Strieter, R.M., Polverini, P.J., Kunkel, S.L., Arenberg, D.A., Burdick, M.D., Kasper, J., et al., 1995. The functional role of the ELR motif in CXC chemokine-mediated angiogenesis. J. Biol. Chem. 270, 27348-27357.

Su, Y.L., Feng, J., Guo, Z.X., Xu, L.W., Wang, J.Y., 2012. Histopathological analysis of golden pompano Trachinotus ovatus infected with Photobacterium damselae subsp. piscicida. Mar. Sci. 36, 75-81.

Sun, J.S., Zhao, L., Sun, L., 2011. Interleukin-8 of Cynoglossus semilaevis is a chemoattractant with immunoregulatory property. Fish Shellfish Immunol. 30, 13621367.

Sun, L.Y., Guo, H.Y., Zhu, C.Y., Ma, Z.H., Jiang, S.G., Zhang, D.C., 2014. Genetic polymorphism of breeding populations of golden pompano (Trachinotus ovatus). South China Fish. Sci. 10, 67-71. 
Van der Aa, L.M., Chadzinska, M., Tijhaar, E., Boudinot, P., Verburg-van Kemenade B.M., 2010. CXCL8 chemokines in teleost fish: two lineages with distinct expression profiles during early phases of inflammation. PLoS One 5, e12384.

Wang, T.T., Song, X.H., Bao, G.M., Zhao, L.X., Yu, X., Zhao, J., 2013. Molecular characterization, expression analysis, and biological effects of interleukin-8 in grass carp Ctenopharyngodon idellus. Fish Shellfish Immunol. 35, 1421-1432.

Zhao, B.B., Katagiri, T., Kondo, H., Hirono, I., 2015. Comparative analysis of two types of CXCL8 from Japanese flounder (Paralichthys olivaceus). Dev. Comp. Immunol. $52,37-47$.

Zhen, P.L., Ma, Z.H., Guo, H.Y., Li, Y.N., Zhang, D.C., Jiang, S.G., 2014. Ontogenetic development of caudal skeletons in Trachinotus ovatus larvae. South China Fish. Sci. 10, 45-50. 